\title{
Genetic polymorphisms adjacent to the CAG repeat influence clinical features at onset in Huntington's disease
}

\author{
I Vuillaume, P Vermersch, A Destée, H Petit, B Sablonnière
}

\begin{abstract}
Objectives-To evaluate possible influences of CCG and $\Delta 2642$ glutamic acid polymorphisms adjacent to the (CAG)n trinucleotide repeat in Huntington's disease gene IT15 on some clinical features (age and symptoms) at onset.

Methods-84 patients and a control group of 68 unaffected relatives were studied. Patients all belonged to a group of affected persons tested for molecular confirmation of Huntington's disease. The length of the CAG repeat sequence in the IT15 gene and the adjacent CCG and $\Delta 2642$ polymorphisms were determined by quantitative polymerase chain reaction.

Results-Two intragenic polymorphisms were studied: (CCG)n and $\Delta 2642$ glutamic acid. Patients were classified firstly according to the size of the CCG rich segment adjacent to the CAG repeat into genotype groups CCG $7 / 7,7 / 8,7 / 9,7 / 10$, and $10 / 10$ and then according to $\Delta 2642$ polymorphism into genotype groups $\mathrm{A} / \mathrm{A}$ (absence of the $\Delta 2642$ deletion), $A / B$, and $B / B$ (presence of the $\Delta 2642$ deletion in respectively one and two alleles). The presence of $\Delta 2642$ mutation was associated with a significant decrease in age at onset, although there was no significant increase in CAG size. A good correlation was found between the (CAG) $)_{n}$ trinucleotide repeat size and the age at onset in patients with genotype AA $\left(r^{2}=0.72\right)$. Within patients of the A/B genotype group however, a significant correlation was found but with a drop of the $r^{2}$ value to 0.44 . No association was found between age at onset and the CCG polymorphism. Although an increased percentage of patients within the $\mathrm{A} / \mathrm{A}$ genotype group had a neurological onset, we found no overall significant association between CCG or $\Delta 2642$ polymorphisms and the nature of symptoms at onset.

Conclusions-The $\Delta 2642$ glutamic acid polymorphism did not affect CAG repeat size nor the nature of symptoms at onset but seems to influence the age at onset in patients with Huntington's disease.

(F Neurol Neurosurg Psychiatry 1998;64:758-762)
\end{abstract}

Keywords: Huntington's disease; clinical features; genotype; polymorphisms

Huntington's disease is an autosomal dominant neurodegenerative disorder affecting about 1 in 10000 people in European popula- tions. It usually presents in adult life with chorea, psychiatric manifestations, and cognitive impairment, leading to progressive dementia. ${ }^{1}{ }^{2}$ This disorder nevertheless shows variation in age and in clinical features at onset. Onset symptoms are neurological, psychiatric/ cognitive, or combined (neurological and psychiatric/cognitive) in respectively $46 \%$ to $59 \%, 23 \%$ to $36 \%$, and $18 \%$ to $30 \%$ of cases. ${ }^{13-5}$ The gene responsible for Huntington's disease was mapped to the tip of the short arm of chromosome 4 in $1983 .{ }^{6}$ The genetic defect associated with the disease was identified in 1993 in a novel gene (IT15) containing a trinucleotide repeat (CAG) $n$ that is expanded in patients with Huntington's disease. ${ }^{7}$ Whereas the number of CAG repeats in IT15 gene ranges from nine to 35 in healthy persons, it ranges from 36 to 121 in patients with Huntington's disease. ${ }^{8}$ The expansion of the (CAG)n repeat thus proved to be a highly specific marker for the diagnosis of Huntington's disease. $^{9}$

After cloning of the Huntington's disease mutation, two genetic polymorphisms were identified close to the CAG tract. The first one was a CCG rich segment downstream to the (CAG)n stretch and the second one was the $\Delta 2642$ glutamic acid polymorphism concerning a deletion of three nucleotides at codon positions $2642-2645$. $^{10-15}$ Both presented polymorphic frequencies on normal and affected chromosomes. Moreover, both were independently associated with differences in CAG repeat length on normal chromosomes, as shown by haplotype analysis. In these studies, a strong linkage disequilibrium was found between the Huntington's disease mutation and alleles at both polymorphic regions: CCG rich length alleles were underrepresented whereas $\Delta 2642$ was overrepresented in Huntington's disease chromosomes.

Numerous attempts to determine correlations between the Huntington's disease repeat length and clinical features have been undertaken. A strong negative correlation $(r=-0.70)$ between age at onset and CAG repeat size was reported overall, the number of CAG repeats in Huntington's disease chromosomes accounting for $50 \%$ of the variation of age at onset. ${ }^{9}{ }^{16-19}$ This correlation could be even stronger as shown recently by Brinkman et al. ${ }^{20}$ As for symptoms at onset, no particular clinical feature correlated with the CAG repeat size. ${ }^{5192122}$ As the variation in age at onset is partially explained by the trinucleotide repeat length, it seemed logical to investigate whether 
Table 1 Distribution of genotypes determined by CCG or 42642 glutamic acid polymorphisms in controls and patients with Huntington's disease

\begin{tabular}{|c|c|c|c|c|c|}
\hline \multirow[b]{2}{*}{ Genotype } & \multicolumn{2}{|c|}{ Control group } & \multicolumn{2}{|c|}{ HD group } & \multirow[b]{2}{*}{$p$ Value overall } \\
\hline & $n$ & $\%$ & $n$ & $\%$ & \\
\hline CCG 7/7 & 19 & 27.9 & 51 & 60.7 & \\
\hline CCG $7 / 8$ & 2 & 3.1 & 2 & 2.4 & \\
\hline CCG $7 / 9$ & 5 & 7.3 & 2 & 2.4 & \\
\hline CCG $7 / 10$ & 37 & 54.4 & 28 & 33.3 & \\
\hline CCG $10 / 10$ & 5 & 7.3 & 1 & 1.2 & \\
\hline Total & 68 & 100 & 84 & 100 & $<0.005$ \\
\hline $\mathrm{A} / \mathrm{A}$ & 57 & 83.8 & 46 & 54.8 & \\
\hline $\mathrm{A} / \mathrm{B}$ & 11 & 16.2 & 34 & 40.5 & \\
\hline $\mathrm{B} / \mathrm{B}$ & 0 & 0 & 4 & 4.7 & \\
\hline Total & 68 & 100 & 84 & 100 & $<0.001$ \\
\hline
\end{tabular}

adjacent polymorphisms (for example, the CCG rich segment and $\Delta 2642$ mutation) also account in part for the clinical heterogeneity of the disease. We therefore examined in 84 independent patients with Huntington's disease the correlation between the age and the nature of symptoms at onset with the CAG repeat size or with any of the genotypes determined by either CCG or $\Delta 2642$ polymorphisms.

\section{Patients and methods}

PATIENTS

Eighty four independent patients with Huntington's disease for whom clinical data were obtained after examination by a member of our neurological department were studied. A control group including 68 unaffected relatives was also assessed. All of these patients gave their informed consent and no one under 18 was sampled unless already affected. Samples from at risk subjects were not examined. Information on age at onset, demographic data, family history, and the nature of symptoms at onset based on the appearance of either neurological, psychiatric, or cognitive symptoms, or even combined symptoms within the first year of onset was obtained from clinical records. Each medical report was screened through a questionnaire recording a list of clinical features at onset. Neurological features included chorea, involuntary movements, clumsiness, dysarthria, unsteadiness, trouble in walking, hypokinesia, rigidity, and epilepsy. Psychiatric features included behavioural abnormalities, depressive or manic symptoms, and other psychotic features. As for cognitive features, memory loss and intellectual impairment were present. Presence or absence of each of these symptoms within the first year of onset was noted for each patient. All clinical assessments were made independently of any information on DNA analysis. Two patients were excluded from the clinical correlation study, due to incomplete clinical data.

DNA ANALYSIS

The length of the CAG trinucleotide repeat was determined from genomic DNA using the polymerase chain reaction (PCR) as originally described ${ }^{6}$ with primers HD1 and HD2, except that amplified products were separated on $5 \%$ denaturing polyacrylamide gels and visualised by silver staining. The PCR products containing the repeated trinucleotide were sized by comparison with sequenced cosmids L191F1 and GUS 72-2130. A reliable sizing of CAG repeats was performed with primers HD1 and HD3 just flanking the CAG repeat as described by Warner et al..$^{23}$ The adjacent CCG repeat track was measured using HD419 and HD482 primers with similar conditions as those described by Andrew et al. ${ }^{11}$ The $\Delta 2642$ glutamic acid polymorphism was detected using previously published methods. ${ }^{24}$ The phase of this polymorphism was not determined. Hence genotypes of controls and patients with Huntington's disease were based either on the CCG or the $\Delta 2642$ polymorphisms. Statistical analysis included the usual paired Student's $t$ test for comparing means of repeat length and of age at onset data. Comparison of genotypes and clinical features were performed using a $\chi^{2}$ test or Yates' corrected $\chi^{2}$ test when necessary.

\section{Results}

DISTRIBUTION OF GENOTYPES DETERMINED BY THE CCG OR THE $\triangle 2642$ POLYMORPHISMS

The CCG segment of seven repeats was overrepresented in Huntington's disease chromosomes (up to $95 \%$ v $60.3 \%$ in normal chromosomes). The unbalanced distribution or linkage disequilibrium between CCG rich segments and the Huntington's disease mutation was highly significant (data not shown). Genotype assessment in controls and patients led to five different groups (table1) according to the number of CCG repeats. The distribution of those genotype groups (CCG 7/7, 7/8, 7/9, $7 / 10$, and $10 / 10$ ) showed a significant difference among patients and controls $(\mathrm{p}<0.005)$. Genotype CCG $7 / 7$ was present in $60.7 \%$ of patients with Huntington's disease whereas only in $27.9 \%$ of controls. On the other hand, genotype $7 / 10$ was more frequent in controls $(54,4 \%)$ than in patients $(33.3 \%)$. Genotype frequency for the $\Delta 2642$ glutamic acid polymorphism was also assessed on controls and patients. Two different genotype groups were identified: group $\mathrm{A} / \mathrm{A}$ characterised by the absence of the deletion, group $\mathrm{A} / \mathrm{B}$ and $\mathrm{B} / \mathrm{B}$ determined by the presence of the deletion on respectively one and two alleles. The distribution was significantly different between patients and controls $(\mathrm{p}<0.001)$. Absence of the $\Delta 2642$ residue (genotype $\mathrm{A} / \mathrm{B}$ ) was found in $16.2 \%$ of controls leading to an allele frequency of $8 \%$ considering that no homozygote (genotype $\mathrm{B} / \mathrm{B}$ ) was found for this deletion. However, the $\Delta 2642$ glutamic acid polymorphism was overrepresented among patients with Huntington's disease, being present in $45.2 \%$ of our Huntington's disease cohort. In this group, we found four patients homozygous for the deletion, therefore calculating a frequency of $25.0 \%$ for the $\mathrm{B}$ allele in patients with Huntington's disease.

RELATION BETWEEN PATIENT GENOTYPE (CCG OR $\triangle 2642$ POLYMORPHISMS) AND AGE AT ONSET CAG repeat size associated with normal and affected chromosomes showed two well separated distributions. The number of CAG repeats varied between 12 and 32 (mean 19.5) 
Table 2 Distribution of age at onset and CAG repeat length and their correlations in each genotype group

\begin{tabular}{llllll}
\hline & & & & \multicolumn{2}{l}{$\begin{array}{l}\text { Correlation age at } \\
\text { onset/CAG }\end{array}$} \\
\cline { 4 - 5 } Genotype & $n$ & Age at onset & CAG & $r$ & $r^{2}$ \\
\hline CCG 7/7 & 51 & $42.8(12.8)$ & $45.6(5.4)$ & -0.744 & 0.55 \\
CCG 7/X & 31 & $43.1(15.0)$ & $45.9(7.3)$ & -0.759 & 0.57 \\
$\mathrm{p}$ & & $>0.90$ & $=0.85$ & $=0.88$ & 0.72 \\
$\mathrm{~A} / \mathrm{A}$ & 45 & $45.5(12.2)$ & $45.0(6.4)$ & -0.848 & 0.74 \\
$\mathrm{~A} / \mathrm{B}+\mathrm{B} / \mathrm{B}$ & 37 & $39.0(14.8)$ & $46.5(5.8)$ & -0.664 & 0.44 \\
$\mathrm{p}$ & & $<0.05$ & $=0.28$ & $=0.05$ & \\
\hline
\end{tabular}

^X contained either 8,9 , or 10 CCG repeats.

in the normal allele, and between 39 and 76 (mean 45.8) in the Huntington's disease allele. In our cohort of patients, age at onset varied between 10 and 70 years (mean 43.0 (SD 13.8)). Age at onset for 63 of them varied between 21 and 59 years, eight patients were juvenile cases and 11 patients had a late onset. The now long established inverse correlation between age at onset and CAG repeat size in patients was confirmed in our study $\left(r=-0.757, \mathrm{p}<10^{-4}\right)$. To evaluate the effect of either the CCG rich or the $\Delta 2642$ glutamic acid polymorphisms, we compared the CAG repeat number and the age at onset in patients carrying different genotypes. Mean age at onset among the different genotype groups according to the CCG polymorphisms varied between 42.8 and 43.1 years, showing no significant difference (table 2). Patients bearing the $\Delta 2642$ glutamic acid mutation $(\mathrm{A} / \mathrm{B}+\mathrm{B} / \mathrm{B}$ genotype group) had an earlier onset of 6.5 years than those belonging to the A/A genotype group (table 2). In this case, the difference in mean age at onset was significant $(\mathrm{p}<0.05)$. In these two genotype groups, log of age at onset correlated significantly with the CAG stretch $(r=-0.848$ and $r=-0.664$ in the $\mathrm{A} / \mathrm{A}$ and in the $\mathrm{A} / \mathrm{B}+\mathrm{B} / \mathrm{B}$ genotype group respectively). However, there was no significant difference between the size of the CAG repeat in these same two genotype groups, suggesting that the differences in mean age at onset could be explained by an indirect influence of $\Delta 2642$ mutation. The difference in age at onset between the $\mathrm{A} / \mathrm{A}$ and $\mathrm{A} / \mathrm{B}+\mathrm{B} / \mathrm{B}$ genotype groups cannot be simply explained by an apparent bias in the distribution of large CAG repeat expansions. In fact, we found a similar distribution of patients with a CAG repeat expansion larger than $50 \mathrm{CAG}$ repeats between the two groups $\left(13.5 \%\right.$ v $20 \%, \chi^{2}=0.98$, $\mathrm{p}=0.45$, data not shown). Moreover, the contribution of the CAG repeat length in the variation of age at onset did not exceed $44 \%$ $\left(r^{2}=0.44\right)$ in patients carrying the $\Delta 2642$ muta-

Table 3 Relation between patient genotype and nature of symptoms at onset

\begin{tabular}{|c|c|c|c|c|}
\hline \multirow[b]{3}{*}{ Onset features } & \multicolumn{4}{|c|}{ Patient genotype } \\
\hline & $C C G 7 / 7$ & $C C G 7 / X$ & $A / A$ & $A / B+B / B$ \\
\hline & \multicolumn{2}{|c|}{$\%$ of patients } & \multicolumn{2}{|c|}{$\%$ of patients } \\
\hline Neurological & 41.2 & 34.5 & 46.7 & 29.7 \\
\hline Psychiatric/cognitive & 27.4 & 24.1 & 22.2 & 27.0 \\
\hline Combined $^{\star}$ & 31.4 & 41.4 & 31.1 & 43.3 \\
\hline p Overall & 0.70 & & 0.45 & \\
\hline
\end{tabular}

${ }^{\star}$ Association of two or three types of symptoms at onset (neurological / psychiatric / cognitive) within the first year of onset. tion whereas it reached $72 \%$ in patients not carrying the deletion $\left(r^{2}=0.72\right)$. On the other hand, the contribution of the CAG repeat size in the variation of age at onset was similar among the two genotype groups CCG $7 / 7$ and CCG $7 / \mathrm{X}$ (X containing either 8,9 , or $10 \mathrm{CCG}$ repeats) determined by the CCG polymorphism. The inverse correlation between CAG size and age at onset was significant with an $r^{2}=0.55$ for patients in genotype group CCG $7 / 7$ and an $r^{2}=0.57$ for patients in genotype group CCG 7/X underlining the absence of any apparent effect of the CCG polymorphism on the correlation between CAG size and age at onset.

RELATION BETWEEN PATIENT GENOTYPE (CCG OR $\triangle 2642$ POLYMORPHISMS) AND THE NATURE OF SYMPTOMS AT ONSET

An assessment was made of the relation between the (CAG)n expansion length and the presentation of clinical features within the first year after onset. The cohort was therefore divided into those who either had neurological symptoms, psychiatric or cognitive manifestations, and into those who had combined symptoms (neurological and psychiatric, or neurological and cognitive, or cognitive and psychiatric symptoms). There was no association between the CAG repeat length and a particular clinical presentation at onset (data not shown).

The influence of either the CCG or the $\Delta 2642$ glutamic acid polymorphism on the nature of symptoms at onset was also examined. There was no overall statistical relation $(p>0.05)$ between the clinical presentation at onset and the different genotypes determined by the CCG rich segment on the one hand, and the $\Delta 2642$ glutamic acid polymorphism on the other hand (table 3 ). Nevertheless, it was noteworthy that $46.7 \%$ of patients with genotype A/A versus $29.7 \%$ of patients with genotype $\mathrm{A} / \mathrm{B}$ or $\mathrm{B} / \mathrm{B}$ had a neurological onset with no other associated symptom. The distribution of genotypes including the various CCG polymorphisms was similar among patients presenting either psychiatric, cognitive, or combined symptoms.

\section{Discussion}

Analysis of the distribution of genotypes determined by the CCG or $\Delta 2642$ polymorphisms in our cohort of 84 patients with Huntington's disease and 68 controls is similar to previous published data. Thus despite the impossibility of assessing the phase of the $\Delta 2642$ polymorphism in our set of patients, the genotype frequency for this polymorphism shows similar deduced allele frequencies in the Huntington's disease gene to those values reported by Almqvist et al, ${ }^{15}$ assuming the fact that a strong disequilibrium exists between $\Delta 2642$ and the Huntington's disease mutation. Regarding the CCG rich segment distribution, our results confirmed what was previously published by other investigators, ${ }^{915}$ showing that expanded $(\mathrm{CAG})_{\mathrm{n}}$ repeats are preferentially associated with a CCG repeat of seven $(96 \%$ of patients have a CCG of seven 
repeats on their affected chromosome, data not shown). This polymorphism was shown to be moderate with the number of repeats varying mostly between seven and 10. Huntington's disease is characterised by a diversity in age at onset as well as in clinical features at onset. The mean age at onset in our set of patients matched what has been published in larger studies. ${ }^{81718}$ Sixty two per cent of our patients were monosymptomatic within the first year after onset (with either neurological, psychiatric, or cognitive features). Our data showed that $38 \%$ of symptoms at onset were neurological, $25 \%$ were psychiatric/cognitive, and $37 \%$ were combined symptoms. The reported data show a slightly higher proportion of combined symptoms compared with previous publications (18 to $30 \%) .^{135}$ Variations in the percentage of onset symptoms were reported among these different studies, reflecting various difficulties in the determination of a precise onset. Firstly, we examined the potential determinism of genetic factors on age at onset. There was a significant difference $(p<0.05)$ in the distribution of ages at onset among patients bearing or not bearing the $\mathrm{B}$ allele. The presence of the $\Delta 2642$ mutation was associated with a significant decrease in the age at onset, although there was no significant increase in the CAG repeat size. Our results match in part what was found by Lucotte et $a l,{ }^{25}$ contradicting results reported by Novelletto et al. ${ }^{13}$ The overall statistical negative correlation of age at onset with the CAG repeat length was verified in our cohort. Nevertheless, the CAG repeat expansion on the Huntington's disease chromosome is not the only factor to influence the function of the Huntington's disease gene, accounting for $56 \%$ of the variation in age at onset of the whole cohort. For patients presenting the $\Delta 2642$ polymorphism, the CAG repeat length only contributed to $44 \%$ of the variation in age at onset versus $72 \%$ of this variation for patients carrying the A/A genotype. This increase in the value of $r^{2}$ in genotype $\mathrm{A} / \mathrm{A}$ cannot be explained by a higher proportion of juvenile cases, as was previously suggested in a group of patients affected with the juvenile form of the disease. ${ }^{26}$ There were actually fewer juvenile patients in this group than in the $\mathrm{A} / \mathrm{B}$ and $\mathrm{B} / \mathrm{B}$ groups (two patients in the $\mathrm{A} / \mathrm{A}$ group versus six patients in the $A / B+B / B$ group). Therefore, presence of the $\Delta 2642$ polymorphism might explain the reduced contribution of the CAG repeat length to age at onset in patients with the $\mathrm{A} / \mathrm{B}$ or $\mathrm{B} / \mathrm{B}$ genotype $\left(r^{2}=0.44\right)$ compared with patients bearing the $\mathrm{A} /$ A genotype $\left(r^{2}=0.72\right)$. Our results show that the $\Delta 2642$ polymorphism may play a part in the determinism in the onset of the disease, which is further supported by the fact that this deletion is enriched in patients with Huntington's disease. This finding suggests that the function of the Huntington's disease gene is influenced not only by the CAG repeat expansion on the Huntington's disease chromosome but also by possible cis-acting specific elements. As the expansion of CAG repeat length is the major factor for the reduction in age at onset, presence of the $\Delta 2642$ mutation may add to the severity of the disease, carriers of this mutation tending to develop the disease earlier. This finding agrees with the opinion that age at onset of Huntington's disease may depend on different factors including the influence of the normal allele $\mathrm{e}^{1727}$ and nonallelic modifying genes. ${ }^{28}$ Genetic components such as genetic imprinting ${ }^{29}$ and aging genes ${ }^{30}$ could be evoked. Other factors including environmental factors may also play a part in the genesis of the affected phenotype. No correlation was found between nature of symptoms at onset and (CAG)n trinucleotide expansion, concurring with results of other studies. ${ }^{5} 192122$ No significant association was found between a specific type of symptom at onset (neurological, psychiatric, or cognitive) and the genetic polymorphisms. However, strikingly, a larger number of patients $(46.7 \%)$ with genotype $\mathrm{A} / \mathrm{A}$ than those with genotype $\mathrm{A} / \mathrm{B}$ or B/B (29.7\%) had neurological features without any psychiatric or cognitive symptoms at onset. This difference was not significant but could indicate a tendency towards a possible association between the presence of genotype $\mathrm{A} / \mathrm{A}$ and the predominance of neurological features at onset. Genetic variants acting cis or trans to the expanded CAG repeat could then influence the phenotypic presentation. The assessment of the $\Delta 2642$ polymorphisms disclosed the potential indirect influence of this genetic polymorphism in the onset of the disease. Whether or not this polymorphism may represent an independent predictor of the age at onset would need further studies of the respective contribution of normal and expanded alleles (deleted or not), on the phenotype; requiring haplotype studies. It could also be relevant to analyse the influence of the genetic polymorphisms on the mode of progression of the disease, as was already reported for the influence on the age at onset. $^{31-33}$ This genotypic study of patients based on the $\Delta 2642$ and CCG-rich segment analysis may contribute to our understanding of the genotype-phenotype relations in Huntington's disease.

We are grateful to Guy Bocquillon, Patrick Devos, Christiane Marzys, and Carole Verlez for their skilful technical assistance and to Isabelle Delalande for her invaluable help with accumulation of clinical data. We are also grateful to A Duhamel (statistician from the CERIM, CHRU de Lille) for help in statistical evaluation. This work was supported by grants from the CHRU of Lille.

1 Hayden MR. Huntington's chorea. New-York: Springer Verlag 1981.

2 Harper PS. Huntington's disease. London: Saunders 1991.

2 Harper PS. Huntington's disease. London: Saunders 1991. Folstein JE. Huntington's disease: a disorder of

4 Di Maio L, Squitieri F, Napolitano G, et al. Onset symptoms in 510 patients with Huntington's disease. $\mathcal{F}$ Med Genet 1993;30:289-92.

5 Claes S, Van Zand K, Legius E, et al. Correlations between triplet repeat expansion and clinical features in Huntington's disease. Arch Neurol 1995;52:749-53.

6 Gusella JF, Wexler NS, Conneally PM, et al. A polymorphic DNA marker genetically linked to Huntington's disease. Nature 1983;306:234-8.

7 The Huntington's Disease Collaborative Research Group. A novel gene containing a trinucleotide repeat that is expanded and unstable on Huntington's disease chromosomes. Cell 1993;72:971-83.

8 Kremer B, Goldberg P, Andrew SE, et al. A worldwide study of the Huntington's disease mutation. $N$ Engl F Med 1994; 20:1401-6. 
9 Andrew SE, Goldberg YP, Kremer B, et al. The relationship between trinucleotide (CAG) repeat length and clinical
features of Huntington's disease. Nat Genet 1993;4:398403.

10 Rubinsztein DC, Barton DE, Davison BCC, et al. Analysis of the huntingtin gene reveals a trinucleotide length polymorphism in the region of the gene that contains two CCG rich stretches and a correlation between decrease age of onset of Huntington's disease and CAG repeat number. Hum Mol Genet 1993;10:1713-5.

11 Andrew SE, Goldberg YP, Theilmann J, et al. A CCG polymorphism adjacent to the CAG repeat in Huntington's disease gene: implications for diagnostic accuracy and predictive testing. Hum Mol Genet 1994;3:65-67.

12 Barron LH, Rae A, Holloway S, et al. A single allele from the polymorphic CCG-rich sequence immediately 3' to the unstable CAG trinucleotide in the IT-15 cDNA shows almost complete disequilibrium with HD chromosomes in the Scottish population. Hum Mol Genet 1994;3: $173-5$.

13 Novelletto A, Persichetti F, Sabbadini G, et al. Polymorphism analysis of the Huntington gene in Italian families affected with

14 Squitieri F, Andrew SE, Goldberg YP, et al. DNA haplotype analysis of Huntington disease reveals clues to the origin and mechanisms of CAG expansion and reasons for geographic variations of prevalence. Hum Mol Genet 1994; 3:2103-14

15 Almqvist A, Spence N, Nichol K, et al. Ancestral differences in the distribution of the $\Delta 2642$ glutamic acid polymorphism is associated with varying CAG repeat lengths on normal chromosomes: insights into the genetic evolution of Huntington's disease. Hum Mol Genet 1995;4:207-14.

16 Duyao M, Ambrose C, Myers R, et al. Trinucleotide repeat length instability and age of onset in Huntington's disease. Nat Genet 1993;4:387-92.

17 Snell RG, MacMillan JC, Cheadle JP, et al. Relationship between trinucleotide repeat expansion and phenotypic
variation in Huntington's disease. Nat Genet 1993;4:3937 .

18 Stine O.C, Pleasant N, Franz ML, et al. Correlation between the onset age of Huntington's disease and the length of the trinucleotide repeat in IT-15. Hum Mol Genet 1993;2: 1547-9.

19 MacMillan JC, Snell RG, Tyler A, et al. Molecular analysis and clinical correlations of the Huntington's disease mutation. Lancet 1993;342:954-8.
20 Brinkman RR, Mezei MM, Theilmann J, et al. The likelihood of being affected with Huntington disease by a
particular age, for a specific CAG size. Am f Hum Genet particular age, for

21 Weigell-Weber M, Schmidt W, Spiegel R. Psychiatric symptoms and CAG expansion in Huntington's disease. $\mathrm{Am} \mathcal{F}$ Med Genet 1996;67:53-7.

2 Zappacosta B, Monza D, Meoni C, et al. Psychiatric symptoms do not correlate with cognitive decline, motor symptoms, or CAG repeat length in Huntington's disease. Arch Neurol 1996;53:493-7.

23 Warner JP, Barron LH, Brock DJH. A new polymerase chain reaction (PCR) assay for the trinucleotide repeat that is unstable and expanded on Huntington's disease chromosomes. Mol Cell Probes 1993;7:235-9.

24 Ambrose CM, Duyao MP, Barnes G, et al. Structure and expression of Huntington's disease gene: evidence against simple inactivation due to an expanded CAG repeat. Somat Cell Mol Genet 1994;20:27-38.

25 Lucotte G, Gerard N, Roubertoux P, et al. Relationships of the 2642 deletion polymorphism $(\Delta 2642)$ in the Huntington gene with the CAG repeat expansion length and age at ton gene with the CAG repeat expansion length and

26 Telenius H, Kremer HPH, Theilmann J, et al. Molecular analysis of juvenile Huntington disease: the major influence on (CAG)n repeat length is the sex of the affected parent. Hum Mol Genet 1993;10:1535-40.

27 Farrer LA, Cupples LA, Wiater P, et al. The normal HD allele, or a closely linked gene influences age at onset of Huntington's disease. Am $\mathscr{f}$ Hum Genet 1993;53:125-30.

28 Farrer LA, Conneally PM. A genetic model for age at onset in Huntington's disease. Am f Hum Genet 1985;37:350-7.

29 Farrer LA, Cupples LA, Kiely DK, et al. Inverse relationship between age at onset of Huntington's disease and paternal Genet 1992;50:528-35.

30 Farrer LA, Conneally PM, Yu PI. The natural history of Huntington's disease: possible role of "aging genes". Am $\mathcal{f}$ Med Genet 1984;18:115-23.

31 Illarioshkin SN, Igarashi S, Onodera $\mathrm{O}$, et al. Trinucleotide repeat length and rate of progression of Huntington's disease. Ann Neurol 1994;36:630-5.

32 Roos RAC, Hermans J, Vegter-van der Vlis M, et al. Duration of illness in Huntington's disease is not related to age at onset. F Neurol Neurosurg Psychiatry 1993;56:98-100.

33 Brandt J, Bylsma FW, Gross R, et al. Trinucleotide repeat length and clinical progression in Huntington's disease. Neurology 1996;46:527-31. 